\title{
Robot control and navigation: ARI's autonomous system
}

\author{
Francesco Ferro ${ }^{1}$, Federico Nardi ${ }^{2}$, Sara Cooper ${ }^{3}$, Luca Marchionni ${ }^{4}$
}

\begin{abstract}
This paper describes ARI robot, the social robot developed by PAL Robotics, highlights its autonomous navigation system framework using Visual SLAM and the potential of combining it with its interaction abilities to achieve safe and robust navigation in human-crowded environments.
\end{abstract}

\section{INTRODUCTION}

Autonomous mobile robots are able to perform different tasks, i.e., human-robot interaction and object handling. In order to perform these tasks, robots must be able to navigate safely and autonomously in different environments that can be dynamic and crowded. Just like humans, robots need information about their surroundings. In particular, the mobile robot has to be able to identify where it is, what is around it and how to travel from the start to the goal point. Avoiding people in crowded environments is especially challenging as they are dynamic, and also needs to consider social aspects of the interaction [1]. Over the past few years, several methods have been presented to approach this matter [2], one of them being the Visual SLAM, which is the technique integrated and used in the ARI robot.

The paper is structured as follows: In Section II we give an overview of ARI main components. In Section III, we describe the autonomous navigation system. Finally, we present conclusions in Section V.

\section{OVERVIEW OF ARI}

ARI is an extension PAL Robotics REEM service robot [3] and as such extends many of its software and general design characteristics. The overall concept of ARI is focused on expressiveness and user engagement. As a social robot, ARI is a platform integrated with AI. The available processing power of the fully ROS based platform allows the user to freely explore the applications of AI and robotics. ARI is an open humanoid platform: its behavior can be easily customized using the provided ROS and REST APIs to effortlessly develop, test on the simulator and deploy applications on the robot.

ARI is designed to engage in high-quality interactions, integrated with Intel core i7 CPU and NVIDIA Jetson TX2 GPU, it is a powerful platform that can be used to develop AI algorithms, and can be equipped with text-to-speech software and human face detection and tracking capabilities. Also equipped with RGB-D and Depth cameras, LEDs, Eyes integrated with LCDs, IMU sensor, microphones array and speakers that can be used for vision and audio capabilities.

\footnotetext{
${ }^{1}$ Francesco Ferro is PAL Robotics' CEO francesco. ferro@palrobotics.com

2 Federico Nardi is a navigation engineer at PAL Robotics Federico.nardiepal-robotics.com
}

In pursuance of performing the tasks that ARI can be used for, such as recognize faces, make gestures, show information on the touchscreen on its chest for user interaction and multimedia content; it can locate itself inside a building and move around while avoiding obstacles in its path.

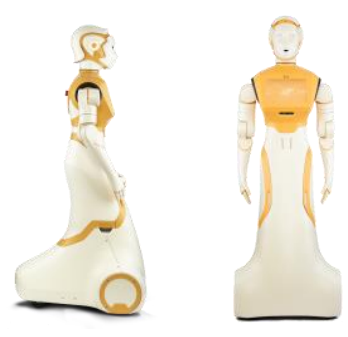

Fig. 1 ARI Robot

\section{AUTONOMOUS NAVIGATION SYSTEM}

When it comes to a robotics platform, it is crucial to choose the most appropriate and convenient navigation system based on the tasks and goals that the robot will be carrying out. The ability to navigate in different environments, in a safe and autonomous manner, capable of avoiding obstacles and managing to conclude a task is vital for any mobile robot. When making this decision, many aspects need to be taken into consideration, such as the robot purpose, platform cost and the energy consumption, amongst others.

In this context, it is relevant to mention two main techniques to tackle the navigation system in any robotic platform: the first one being the LiDAR method, which uses a 2D laser range finder to measure distances to objects in the scene $[4,5]$. The LiDAR, which stands for Light Detection and Ranging, is able to do this measurement by illuminating an object with multiple transceivers that will emit pulsed light able generate data to determine the distance and position of the certain object.

The second one system is the Visual SLAM (simultaneous localization and mapping) method: with a camera that uses 3D vision, this system is able to conduct mapping and location [6], highly beneficial for the user as it is a more cost-effective and accessible option; opposed to the LiDAR navigation system is significantly less expensive.

REEM, its predecessor, has been deployed at crowded areas like shopping malls and exhibition centers to safely navigate around humans (see Fig 2). It uses the LiDAR method for navigation.

\footnotetext{
Sara Cooper is a robotics software engineer at PAL Robotics sara.cooperepal-robotics.com

4 Luca Marchionni is PAL Robotics' CTO luca.marchionniepal-robotics.com
} 


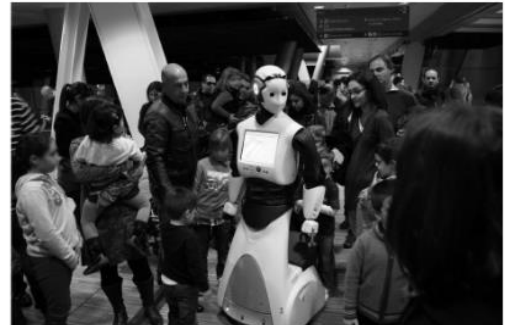

Fig. 2 REEM acting as a guide in a crowded event at La Caixa Science Museum Barcelona 2012

To ensure safety in crowded areas ARI includes several sensors for detecting static and dynamic obstacles. In contrast to REEM, it is provided with an autonomous navigation system based on Visual SLAM [7] to perform mapping and localization using the frontal RGB-D camera of the torso. This system works by detecting key points or features from the camera input and recognizing previously seen locations in order to create a map and localize. The map obtained is represented as an Occupancy Grid Map (OGM) that can later be used to make the robot localize and navigate autonomously in the environment using move_base.

The navigation software provided with ARI can be seen as a black box with the inputs and outputs shown in Figure 3, based on ROS (Robotics Operating System).

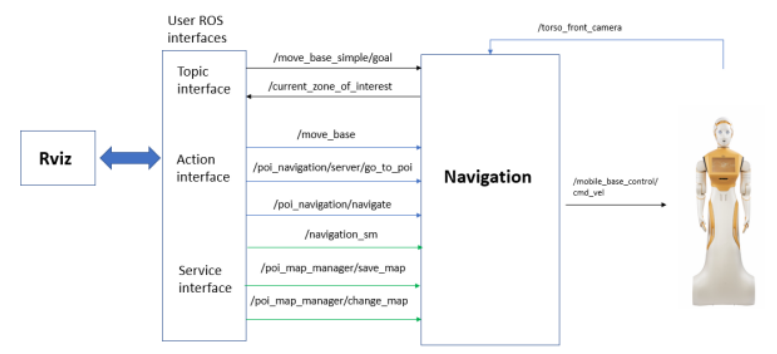

Fig. 3 - ARI navigation system interface

As can be seen, the user can communicate with the navigation software using ROS actions and services. Note that $\mathrm{RViz}^{5}$ also can use these interfaces to help the user perform navigation tasks.

By using the Visual SLAM navigation system, ARI is able to perform autonomous mapping, localization and obstacle avoidance in different environments that could be crowded and dynamic, experiencing modifications in a short time notice.

The final system to be used on ARI is a combination of the back stereo camera with ORB-SLAM for localization and the front depth camera (point cloud camera) for obstacle avoidance. The strength of this approach is that the localization shouldn't be affected by the people in front of the robot while navigation.

The strength of this approach is that the localization shouldn't be affected by the people in front of the robot while navigation. From PAL Robotics experience with REEM this is important as users tend to place themselves in front of the robot to interact with it. By using a back camera, the robot

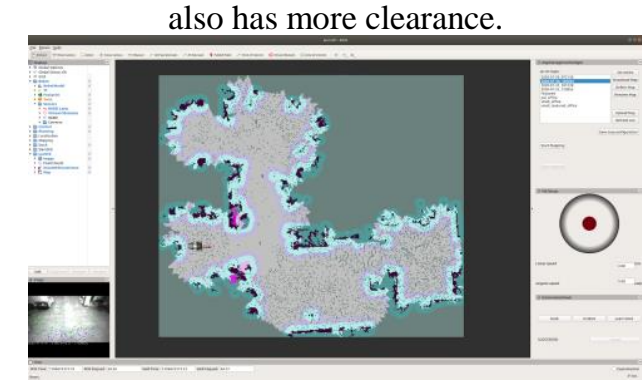

Fig. 4 - Example of the ARI's autonomous navigation

Further work will also add components of REEM such as adapting its speed based on distance to nearest obstacles and based on proxemic interpersonal distances [1], as well as use its multi-modal behaviour to indicate its intention to the surrounding people, important for human-aware navigation [1]. For that ARI can use its LEDs, torso touch-screen and speech synthesis, for instance, to tell people where the robot will go next, or indicate that it cannot move due to an obstacle. By using its head RGB camera people and object detection algorithms already implemented will provide essential for adapting to the dynamic environment. As part of H2020 SPRING project ${ }^{6}$, that PAL Robotics is participating in, ARI's navigation abilities will be exploited and advanced to use it as a receptionist of a hospital.

\section{CONCLUSION}

This paper described the autonomous navigation system of ARI, the high-performance robotic platform created by PAL Robotics. ARI has been designed for a wide range of multimodal expressive gestures and behaviors, making it a social robot suitable for Human-Robot-Interaction, perception, cognition and navigation, and can be a platform for research in navigation in crowded environments.

\section{REFERENCES}

[1] Kruse, T., Pandey, A. K., Alami, R., \& Kirsch, A. (2013). Human-aware robot navigation: A survey. Robotics and Autonomous Systems, 61(12), 1726-1743.

[2] Cadena, Cesar, et al. "Past, present, and future of simultaneous localization and mapping: Toward the robust-perception age." IEEE Transactions on robotics 32.6 (2016): 1309-1332.

[3] L. Marchionni, J. Pages, J. Adell, J. R. Capriles, and H. Tome, "Reem service robot: how may I help you?" in Proc. of the Int. WorkConference on the Interplay between Natural and Artificial Computation, IWINAC, Palma de Mallorca, Spain, June 2013, pp. 121130 .

[4] Durrant-Whyte, Hugh, and Tim Bailey. "Simultaneous localization and mapping: part I." IEEE robotics \& automation magazine 13.2 (2006): 99-110.

[5] Bailey, Tim, and Hugh Durrant-Whyte. "Simultaneous localization and mapping (SLAM): Part II." IEEE robotics \& automation magazine 13.3 (2006): 108-117.

[6] Bonin-Font, Francisco, Alberto Ortiz, and Gabriel Oliver. "Visua navigation for mobile robots: A survey." Journal of intelligent and robotic systems 53.3 (2008): 263.

[7] Mur-Artal, R., Montiel, J. M. M., \& Tardos, J. D. (2015). ORB-SLAM: a versatile and accurate monocular SLAM system. IEEE transactions on robotics, 31(5), 1147-1163. 